\title{
Desarrollo de la primera etapa del sistema de emisión de títulos profesionales digitales conforme a los lineamientos de la Secretaría de Educación Pública
}

\section{Development of the first stage of the system for issuing Digital Degree Certificate in accordance with the guidelines of the Ministry of Public Education}

\author{
MORALES-OROZCO, David†*, ALCÁNTAR-ORTIZ, Patricia, VEGA-OLVERA, Gustavo Ivan y \\ VEGA-FLORES, Patricia
}

Instituto Tecnológico Superior del Sur de Guanajuato

ID $1^{\text {er }}$ Autor: David, Morales-Orozco / ORC ID: 0000-0002-3193-4709

ID $1^{\text {er }}$ Coautor: Patricia, Alcántar-Ortiz

ID $2^{\text {do }}$ Coautor: Gustavo Ivan, Vega-Olvera / ORC ID: 0000-0002-0172-5383

ID $3^{\text {er }}$ Coautor: Patricia, Vega-Flores / ORC ID: 0000-0002-1375-5803

DOI: $10.35429 / J U M .2019 .8 .3 .12 .16$

Recibido 10 de Marzo, 2019; Aceptado 30 de Junio, 2019

\section{Resumen}

Se describen los resultados de la primera etapa del proyecto Sistema de emisión de títulos profesionales digitales, que tiene como objetivo generar una herramienta que permita la emisión de títulos digitales de acuerdo a los nuevos lineamientos de la Secretaría de Educación Pública (publicados en el diario oficial de la federación) en el Instituto Tecnológico Superior del sur de Guanajuato. La metodología del presente proyecto se propone mediante el uso del ciclo de vida basado en los modelos iterativo e incremental, que permitirá dividir el proyecto en iteraciones que generen incrementos de valor, con el fin de estabilizar el sistema en etapas tempranas. Al emitir los títulos digitales se quiere aprovechar y potenciar el sistema integral de control escolar que se tiene actualmente en la institución con lo cual se tendrá una mejora en el servicio a nuestros estudiantes al no depender de terceros en la emisión del título electrónico esto nos permitiría agilizar la atención al estudiante y conocer en todo momento el estado actual del proceso de emisión del título digital. En la primera etapa se tienen definidos los requerimientos, el diseño y el prototipo no funcional, con la primera versión de generación del XML correspondiente al título.

Software, Títulos profesionales digitales, Egresados

\begin{abstract}
The results of the first stage of the project System for issuing digital professional titles are described, which aims to generate a tool that allows the issuance of Digital Degree Certificate according to the new guidelines of the Ministry of Public Education (published in the official gazette of the federation) in the superior technological institute of southern Guanajuato. The methodology of this project is proposed through the use of the life cycle based on the iterative and incremental models, which will allow the project to be divided into iterations that generate value increases, in order to stabilize the system in early stages. When issuing the digital titles, we want to take advantage and enhance the comprehensive system of school control that is currently in the institution, which will improve our service to our students by not relying on third parties in the issuance of the electronic title that would allow us Streamline student attention and know at all times the current status of the digital title issuance process. In the first stage the requirements, design and non-functional prototype are defined, with the first generation version of the XML corresponding to the title.
\end{abstract}

Software, Digital Degree Certificate, Graduates

Citación: MORALES-OROZCO, David, ALCÁNTAR-ORTIZ, Patricia, VEGA-OLVERA, Gustavo Ivan y VEGAFLORES, Patricia. Desarrollo de la primera etapa del sistema de emisión de títulos profesionales digitales conforme a los lineamientos de la Secretaría de Educación Pública. Revista de Gestión Universitaria. 2019. 3-8: 12-16

$\dagger$ Investigador contribuyendo como primer autor. 


\section{Introducción}

Actualmente se está llevando un cambio paulatino en la manera en que tradicionalmente se gestionaba la documentación oficial en la que todo el manejo de la misma se tenía en un formato físico debidamente firmado y sellado, actualmente se está haciendo una transición de este modelo a tener la información en electrónico con las debidas autorizaciones digitales es decir firmas electrónicas y sellos digitales que permiten verificar la autenticidad de los documentos electrónicos.

En estos momentos uno de los cambios se está realizando en la expedición de títulos universitarios, en lo que las instituciones de educación superior deben tener una manera de generar dichos títulos electrónicos de manera electrónica para que puedan ser utilizados en un momento posterior para obtener la cedula electrónica.

Por lo anterior es que el Instituto Tecnológico Superior del Sur de Guanajuato (ITSUR), está desarrollando un sistema informático que facilite la realización del título electrónico para los alumnos egresados de la institución y agilizar el trámite que conlleva a los egresados realizar este proceso.

Es de destacar que el ITSUR, en su momento llego a tener la acreditación de CMMi Dev nivel 3 que es un modelo internacional para el desarrollo de software lo que nos permite sacar provecho de esta experiencia que se tiene en el desarrollo de sistemas para desarrollar un producto de calidad que cumpla con las especificaciones requeridas de este importante tramite de nuestros estudiantes que representa la culminación de años de esfuerzo en sus estudios universitarios.

\section{Antecedentes}

A lo largo de estas últimas tres décadas, la firma electrónica, un concepto jurídico y que es un equivalente electrónico al de la firma manuscrita, ha ido tomando fuerza debido a los avances tecnológicos de los dispositivos electrónicos y a la evolución de los mecanismos de seguridad computacional (algoritmos). Algunos ejemplos de estos dispositivos electrónicos o técnicas son: firma biométrica, firma con lápiz electrónico, a través de pantallas táctiles, uso de usuario y contraseña.
Un uso importante de la firma electrónica es tener la capacidad de firmar documentos a través de medios electrónicos, ya mencionados.

La firma electrónica se clasifica en básica y avanzada, (MM. Morales-Sandoval, 2013) menciona en su reporte técnico que la firma electrónica básica involucra medios electrónicos que no garantizan los servicios de no repudio, ni los servicios de integridad. Por otro lado describen que la firma electrónica avanzada o firma digital se debe entender como tal, una firma que a traves de un certificado digital emitido por una entidad de certificación acreditada, incorpore una serie de datos electrónicos que identifican y autentifican al firmante a través de la asignación de una llave pública y otra privada en base a los parámetros de la criptografía asimétrica (o también conocida como de llave pública).

La firma electrónica avanzada se usa para autenticar la identidad de quien envía un mensaje o quien firma un documento electrónico, así como asegurar que el contenido original del mensaje o del documento electrónico que ha sido enviado no ha sido modificado, también son fáciles de transportar y no pueden imitarse, puede aplicarse a cualquier tipo de información electrónica.

En México se creó la Ley de Firma Electrónica Avanzada (LFEA) la cual fue publicada en el diario oficial de la federación el 11 de enero de 2012. Esta ley establece que la Firma Electrónica Avanzada tiene los mismos efectos jurídicos que la firma autógrafa, garantiza el contenido del documento firmado y no es repudiable. La Secretaría de Economía, a través de la Secretaria de Hacienda y Crédito Público, es la encargada de proveer y acreditar los servicios de Firma Electrónica Avanzada.

El uso de la firma electrónica avanzada en México inicio con la Secretaria de Administración Tributaria (SAT), sin embargo su uso comenzó a trascender en distintos trámites burocráticos de gobierno, en distintas secretarias y en todos los niveles de gobierno, federal, estatal y municipal.

En Julio de 2018 la Secretaria de Educación Pública (SEP) dio a conocer el proyecto que permite expedir cedulas profesionales electrónicas y el registro de títulos profesionales electrónicos. 
También público en su sitio oficial que a partir del 1 de Octubre de 2018, el registro de título y expedición de cédula profesional, a cargo de la Dirección General de Profesiones (DGP) de la SEP, es en línea a través del portal www.gob.mx/cedulaprofesional, en la cual se describe el proceso y los requisitos necesarios para la expedición de cédulas profesionales, entre ellos la firma electrónica avanzada (e.firma) del profesionista.

\section{Marco Teórico}

En Julio del 2018, cuando se dio a conocer la expedición de cédula profesionales electrónicas por parte de la DGP, se presento también el concepto de Títulos Profesionales electrónicos los cuales deben ser generados y firmados digitalmente por las instituciones de educación superior para que enseguida estos sean registrados y validados ante la dirección general de profesiones, todo a través de Internet. El proceso de expedición de títulos profesionales electrónicos es el objeto del presente proyecto.

La encomienda que dio la SEP a las instituciones superiores viene acompañado de un proceso y una plataforma web para el registro y validación de títulos profesionales electrónicos, dejando la responsabilidad de generar y firmar digitalmente el título profesional electrónico.

Los requisitos técnicos para generar
títulos profesionales de describen a
continuación:

Obtener la firma electrónica avanzada (e.firma) de las personas firmantes del título.

- Estructura del archivo electrónico que representa el título.

Credenciales para acceder a la plataforma web de la DGP que permite registrar y validar títulos electrónicos.

La firma electrónica avanzada que es emitida por el SAT, está basado en certificados digitales con Infraestructura de llave pública (PKI) y criptografía de llave pública. Este mecanismo garantiza que en el caso de sufrir variaciones en la firma y/o gestión de documentación electrónica, la responsabilidad es del usuario, ya que al tener esta firma bajo su control exclusivo, el usuario es por tanto el responsable último de todos los procesos asociados a la misma.
La DGP determino la estructura del archivo electrónico que representa el título. El archivo está estructurado a través de lenguaje extensible de marcas (XML) y fue proveído por la misma dirección.

Las credenciales para acceder a la plataforma web de la DGP que permite registrar y validar títulos electrónicos es un trámite que debe gestionar cada Institución Superior ante la dirección general de profesiones.

El Proceso de liberación en ambientes de pruebas y producción, anexo 1, describe el proceso para la generación, registro y validación de títulos electrónicos. En este proceso también se describe como realizar la implementación de lo títulos digitales en fase de pruebas para después pasar a un ambiente de implantación en producción.

El Instituto Tecnológico de Celaya es una de las instituciones del Tecnológico Nacional de México (TecNM) que ya tiene una solución, una plataforma web, que puede ser adaptable a instituciones del TecNM, sin embargo, esto involucraría un gasto por cada título expedido en esa plataforma. Existen otras universidades, fuera del TECNM, que también han emprendido y desarrollado soluciones hechas a la medida de cada institución y que por ende no es posible adaptarlos en cualquier otra Institución.

El Instituto Tecnológico Superior del Sur de Guanajuato (ITSUR), a través del cuerpo académico "Desarrollo de aplicaciones bajo metodologías de Ingeniería de Software" ha decidido emprender el desarrollo de una plataforma web que permita generar, registrar, validar y emitir títulos electrónicos, esto a pesar de que otras instituciones de Educación Superior ya tienen desarrollada una solución.

La decisión está basada bajo el contexto de las soluciones que ya se encuentran, la capacidad técnica con que cuenta cuerpo académico para el desarrollo de software y la necesidad de integrar esta plataforma al Sistema Integral de Control Escolar (SICE) del ITSUR.texto 


\section{Impacto o beneficio}

Con el presente desarrollo se estaría cumpliendo con las nuevas disposiciones con respecto a los títulos profesionales, para que puedan ser registrados por la Dirección General de Profesiones, deben de ser recibidos en forma electrónica, conforme al estándar que ésta publique en el Diario Oficial de la Federación.

Es por este motivo que a la fecha es una prioridad del instituto indicada por director general de la misma, el tener una forma de emitir los títulos digitales de los estudiantes, para lo cual se quiere aprovechar y potenciar el sistema integral de control escolar (SICE) que se tiene actualmente en la institución y que fue desarrollado completamente por nosotros, con lo cual se tendría una mejora en el servicio que ofrecemos a nuestros estudiantes al no depender de terceros en la emisión del título electrónico, ya que la información base para generar el título ya está en nuestro sistema. Al no tener que usar un intermediario en el proceso entre generar nuestra información y generar el título, nos permitiría agilizar la atención al estudiante y conocer en todo momento el estado actual del proceso de emisión del título digital.

Adicionalmente se tiene contemplado que se tendría un ahorro económico al no tener que estar pagando a un tercero la realización de este proceso lo cual actualmente sería: $\$ 10,000.00$ pesos por semestre lo cual incluye un costo aproximado de $\$ 200$ pesos por título de 50 alumno por semestre que requerirán este servicio en la institución. De manera colateral, esta plataforma permitirá establecer convenios con las demás instituciones que tienen instalado el SICE, que actualmente incluye a 5 tecnológicos descentralizados y una escuela normal para que ellos puedan igualmente emitir los títulos digitales en sus instituciones aprovechando la infraestructura que tienen actualmente.

\section{Metodología}

La metodología del presente proyecto se mediante el uso del ciclo de vida ITSUR basado en modelos iterativo e incremental, que permitirá dividir el proyecto en iteraciones que generen incrementos de valor en la aplicación, con el fin de estabilizar la aplicación en etapas tempranas, identificando y corrigiendo defectos de los incrementos después de ponerlos en producción.
En este trabajo se describe hasta la fase 3 la cual actualmente es la que está en desarrollo del proyecto.

El ciclo de vida a usar para cada iteración plantea el desarrollo de diversas etapas o fases, las cuales se especifican a continuación:

Fase 1. Especificar los requerimientos de la plataforma web.

En esta fase se llevó a cabo el análisis y la especificación de los requerimientos de la plataforma de acuerdo a las necesidades previamente detectadas para la plataforma y se llevará a cabo mediante el uso de casos de uso, se puede ver un ejemplo de caso de uso en la figura 1 .

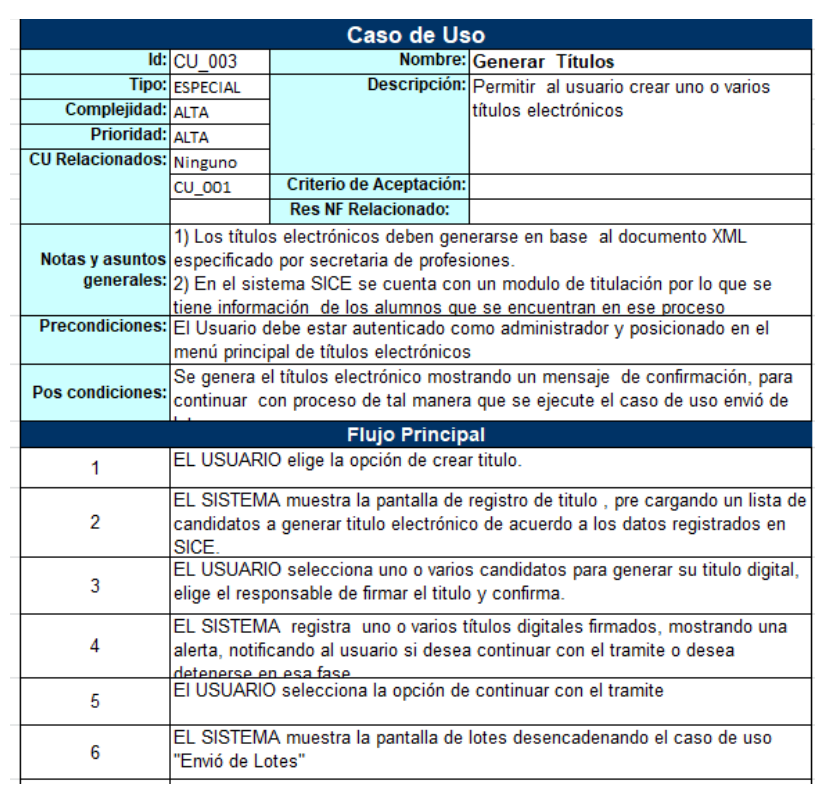

Figura 1 Ejemplo de caso de uso

Fuente: Elaboración Propia

Fase 2. Diseño de la solución.

En esta fase se eligió desarrollar sobre la plataforma .NET sobre la cual se desarrollará la aplicación, tomando como base SICEnet, la cual esta ASP.net.

Se comenzó a homologar los catálogos de la DGP con los catálogos del Sistema Integral Control Escolar del ITSUR.

En este punto se está revisando la comunicación con la DGP requiere que la comunicación con su sistema se realice mediante un bus de servicios web para el intercambio de datos. 


\section{Fase 3. Construcción.}

La integración de los diversos componentes construidos, están siendo integrados mediante el uso de herramientas de trabajo colaborativo como GitHub.

\section{Resultados}

Hasta la fecha de hoy, el $40 \%$ de avance. Las actividades que se han realizado son propias de las fases de análisis, diseño y programación. En la fase de análisis y diseño se realizó una revisión entre pares de los casos de uso, realizando los ajustes derivados de dicha revisión. Se hizo una reestructuración de los requerimientos funcionales, así como de los casos de uso, para reflejar un mejor flujo de la creación y monitoreo de los títulos.

En cuanto a la programación, se tiene implementada la arquitectura del sistema que incluye: backend con algunos DAOs (Data Access Object) y servicios web. El repositorio del código del proyecto preparado para ser versionado con Git.

Se ha desarrollado el prototipo que genera el xml del título que es la base del sistema. En cuanto a cuestiones administrativas se inició el trámite de envió de datos del director ante DGP, para hacer oficial quien firmará digitalmente los títulos.

En la figura 2, se puede apreciar la pantalla para generar títulos, ventana desde la cual se puede iniciar el trámite de un título digital individual.

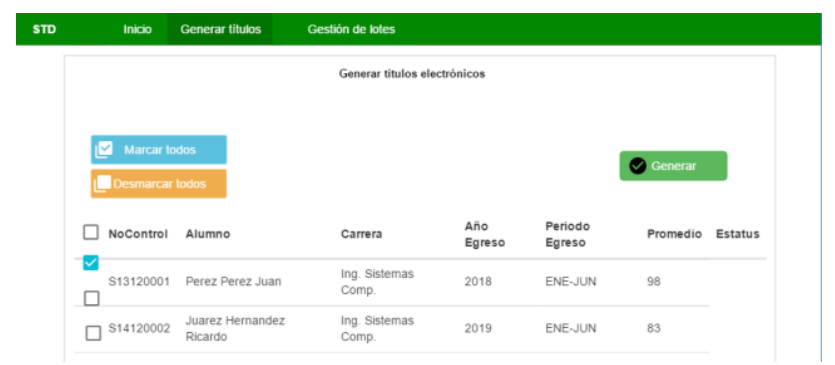

Figura 2 Pantalla generar títulos Fuente: Elaboración Propia

Por último en la figura 2 se puede ver la pantalla que será más utilizada en el sistema la cual permite generar lotes de títulos digitales y dar el seguimiento del lote para revisar el estado en el que se encuentra de acuerdo al proceso establecido.

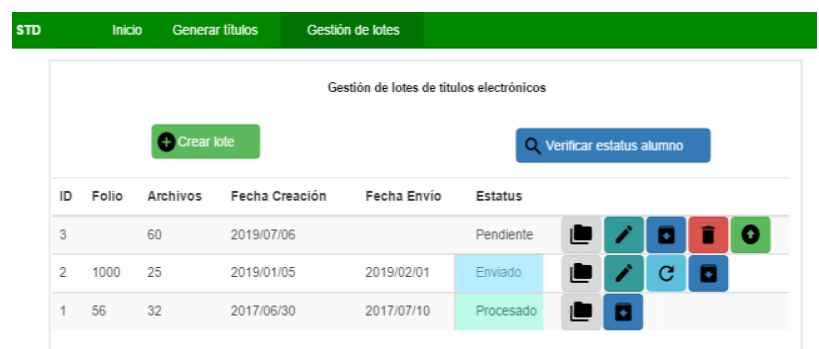

Figura 3 Pantalla gestión de lotes

Fuente: Elaboración Propia

\section{Agradecimiento}

Agradecemos al Tecnológico Nacional de México por el apoyo recibido para el desarrollo del presente proyecto.

\section{Conclusiones}

El desarrollo del proyecto ha sido de impacto al interior de los cuerpos académicos del ITSUR, ya que este es el primer proyecto desarrollado con apoyo del TECNM del cuerpo académico con clave ITESSGTO-CA-3, esto permite que el cuerpo académico vaya avanzando hacia una consolidación del mismo al generar productos que impacten y sean de utilidad.

El trabajo que queda por delante es un reto debido a que la mayoría de la programación del sistema aún queda por realizarse lo que obliga a no perder el ritmo de trabajo realizado hasta al momento para poder terminar en tiempo $\mathrm{y}$ forma con el objetivo del proyecto.

\section{Referencias}

México, G. d. (2018). Ayuda de Títulos Electrónicos. Recuperado el 2019, de https://www.sep.gob.mx/es/sep1/Ayuda_de_Tit ulos_Electronicos

MM. Morales-Sandoval, A. D.-P.-P. (2013). Firma electrónica: concepto y requerimientos para su puesta en práctica. Reporte Técnico Número 1. Tamaulipas: CINVESTAVTamaulipas.

Vega Olvera, G. I., Martínez López, F. J., \& Alcántar Ortiz, P. (2015). Sistema Integral de Control Escolar. Anfei digital, 7.

México, G. d. (2018). Cedula Profesional en México. Recuperado el Enero de 2019, de https://www.gob.mx/cedulaprofesional 